\title{
Clinician-Induced (Iatrogenic) Damage Incurred during Human Fertility Treatment: Detrimental Effects upon Gamete and Embryo Viability and the Potential for Epigenetic Risk
}

\author{
Junaid Kashir, Suseela Yelumalai, Celine Jones and Kevin Coward \\ Nuffield Department of Obstetrics and Gynaecology, Level 3, Women's Centre, John Radcliffe Hospital, Headington, Oxford, OX3 9DU, UK
}

Human infertility affects approximately $10 \%$ of couples worldwide and has led to the development of a suite of clinical laboratory techniques designed to combat such conditions, collectively referred to as 'Assisted Reproductive Technology' (ART) [1]. Such methodology has subsequently resulted in the birth of more than 4.6 million babies worldwide. Infertility may arise as a result of individual male or female factors, a combination of both, or for unknown reasons (idiopathic infertility) [2,3]. While clinical and laboratory aspects of ART have been significantly refined since their first successful clinical application, global pregnancy and live birth rates from ART rarely exceed $\sim 40 \%$ [3]. Indeed, successful pregnancy and delivery rates from conventional ART techniques such as in vitro fertilisation (IVF) or Intracytoplasmic Sperm Injection (ICSI) may be as low as $22.4 \%$ and $23.3 \%$ respectively [3]. As the number of couples seeking infertility treatment increases, it is crucial to develop new techniques and protocols to enhance the success of ART [4].

During ART, gametes and embryos are, by necessity, removed from their highly specialised natural environment, raising the possibility that extraction, storage, and in vitro fertilisation within an artificial environment may result in adverse clinician-derived (iatrogenic) changes, thus compromising quality and viability. While there are many potential causes, it is possible that clinical interventions may in fact represent contributory factors for the relatively poor success rates of ART. Characterising the precise source and extent of such effects would provide a valuable opportunity to modify and improve existing protocols [4-7]. For example, there is significant debate surrounding the potential effects that ART techniques, such as sperm swim-up or density gradient washing, routinely implemented to separate sperm from the seminal plasma of male patients to select the healthiest sperm for downstream processing, may be exerting upon sperm viability [8]. Such methodology can lead to the production of Reactive Oxygen Species (ROS) within sperm [8-12], or induce DNA damage $[13,14]$. Another cause for concern is the routine use of preservative methodology such as cryopreservation, which involves the freezing of cells or tissues to sub-zero temperatures (commonly $\sim-196^{\circ} \mathrm{C}$ ) using liquid nitrogen [6]. The extremely low temperatures involved in cryopreservation prevent biological activity such as cell death, enabling gametes and blastocysts to be preserved until a much later date. However, cryopreservation is a harsh and damaging procedure which can cause extensive cellular damage to a living biological tissue or cell, and may alter or reduce levels of key sperm factors essential for sperm function [4-7].

Approximately $75 \%$ of human embryos produced in vitro may undergo some form of embryo fragmentation (a chaotic division of embryonic cells) [15,16]. It is unclear whether this form of fragmentation arises inadvertently due to culture conditions and follicular stimulation in ART, or represents a normal characteristic of human embryo development [17]. Indeed, it is possible that factors such as culture conditions, prolonged exposure to oxygen, sunlight, specific wavelengths of light (e.g., ultra-violet), inadequate storage, or the absence of a dynamic in vivo environment incorporating cilial movement and muscle contraction, contributes to such phenomena $[18,19]$. The use of higher embryo:culture ratios have been proposed as a possible avenue leading to improved success rates, due to the 'co-operative interaction' of growth factors released in a paracrine or autocrine manner [19-22], or the dilution of toxic factors [22-24].

However, laboratories have been slow to implement these techniques owing to the practice of culturing embryos individually for identification purposes [5]. Currently, laboratories are responsible for deciding which culture techniques they prefer to adopt, despite evidence that environmental factors affect not only ART success, but also the size and weight of the developing zygote. A consensus of opinion is now required as, alongside possible physical damage to cells affecting implantation success rates, environmental factors may also cause genetic/epigenetic defects which could affect the future health of the child and, by inheritance, future generations. Characterisation and understanding of such phenomena is currently very poor, although recent research indicates significant differences between different ART methodologies.

Research focussed upon oocyte activation, a fundamental step in fertilisation which results in the initiation of embryogenesis in response to oscillatory changes in intracellular calcium $\left(\mathrm{Ca}^{2+}\right)$ [25], has revealed that the spatial distribution and duration of $\mathrm{Ca}^{2+}$ oscillations and associated gene expression profiles were not equivalent in mouse oocytes following ICSI compared to those following IVF. $\mathrm{Ca}^{2+}$ oscillation frequency and amplitude influence protein expression profiles in mouse zygotes, development/transplantation rates of rabbit embryos, and are directly responsible for cell-cycle progression rates [25]. Furthermore, following fertilisation, the paternal genome is subject to active demethylation, while the maternal genome is passively demethylated during subsequent cleavage divisions. Worryingly however, histone methylation patterns differ between IVF and ICSI in both mice and in humans, while the demethylation dynamics of the paternal rat genome at the pronuclear-stage is impaired following IVF/ ICSI [26-28]. Understanding the mechanisms underlying methodology

${ }^{*}$ Corresponding author: Kevin Coward, Nuffield Department of Obstetrics and Gynaecology, Level 3, Women's Centre, John Radcliffe Hospital, Headington, Oxford, OX3 9DU. UK, E-mail: kevin.coward@obs-gyn.ox.ac.uk

Received November 08, 2012; Accepted November 09, 2012; Published November 12, 2012

Citation: Kashir J, Yelumalai S, Jones C, Coward K (2012) Clinician-Induced (latrogenic) Damage Incurred during Human Fertility Treatment: Detrimental Effects upon Gamete and Embryo Viability and the Potential for Epigenetic Risk. Human Genet Embryol 1:e105. doi:10.4172/2161-0436.1000e105

Copyright: ( $) 2012$ Kashir J, et al. This is an open-access article distributed unde the terms of the Creative Commons Attribution License, which permits unrestricted use, distribution, and reproduction in any medium, provided the original author and source are credited. 
Citation: Kashir J, Yelumalai S, Jones C, Coward K (2012) Clinician-Induced (latrogenic) Damage Incurred during Human Fertility Treatment: Detrimental Effects upon Gamete and Embryo Viability and the Potential for Epigenetic Risk. Human Genet Embryol 1:e105. doi:10.4172/2161$0436.1000 \mathrm{e} 105$

such as IVF and ICSI, and the potential effect they may exert upon early embryogenesis is vital, as current IVF/ICSI procedures may inadvertently predispose developing embryos to epigenetic risk. Male infertility accounts for $19-57 \%$ of infertility cases, and often requires the use of ICSI to provide the best chance of fertilisation. Despite this revolutionary approach, $\sim 1-5 \%$ of ICSI cycles are still known to fail. Considering that approximately $52 \%$ of all IVF cycles in the UK involve ICSI, thus exposing over 1000 couples annually in the UK alone to potentially deleterious effects $[2,25]$.

A significant issue underlying the potential effect of ART methodology upon resultant offspring has been that such clinical interventions have only been in routine use for the past three decades, with infants born as a result of such methodology only now reaching maturity. Numerous reports now indicate an increased incidence of rare imprinting disorders following the implementation of ART, including Beckwith-Wiedemann, Angelman, and Silver-Russell syndromes. Furthermore, several studies now suggest that epigenetic alterations at imprinted loci occur during the in vitro manipulation of gametes following both IVF and ICSI [29-31]. Evidence also suggests that the association between imprinting disorders and ART may be restricted to syndromes involving hyper-methylation as well as hypo-methylation, with imprinting changes occurring on both parental alleles [29]. These findings may indicate that a proportion of the developmental defects or abnormal growth patterns reported in progeny arising from ART may be associated with a wide combination of epigenetic alterations at imprinted regions [29-31].

The extensive development of ART has not only revolutionised the clinical treatment of infertility, but has also facilitated significant advances in our wider understanding of human reproduction. However, pregnancy and birth rates resulting from such technology remain frustratingly low. Modifications of current ART protocols and the development of novel techniques associated with advancement in science and technology are essential in order to provide improved clinical and diagnostic tools for patients. Recognising the potential for iatrogenic damage upon gametes and embryos during ART will facilitate the improvement, development, and introduction of modified ART protocols which are highly likely to improve success. Furthermore, recent studies implicate iatrogenic alterations in the signalling pathways regulating fertilisation and early zygote development in response to IVF and ICSI, which may result in alterations to embryonic gene expression, as well as increased epigenetic risk. Such phenomena is currently poorly understood, and seems increasingly likely to represent the causative factors underlying conditions which may manifest as disease, not only in young children but perhaps in adults as well $[32,33]$. Considering that the implementation of ART is expanding rapidly worldwide, such questions highlight the urgent need to conduct long-term international studies on ART treatment, and their possible association with the prevalence of imprinting disorders. This is particularly prudent when one considers that aside from imprinting disorders, other pathologies such as general growth or various cancers may also be influenced by ART.

\section{References}

1. Gnoth C, Godehardt D, Godehardt E, Frank-Herrmann P, Freundl G (2003) Time to pregnancy: results of the German prospective study and impact on the management of infertility. Hum Reprod 18: 1959-1966.

2. HFEA: Latest UK IVF Figures-2009 and 2010

3. Nygren KG, Sullivan E, Zegers-Hochschild F, Mansour R, Ishihara O, et al. (2011) International Committee for Monitoring Assisted Reproductive Technology (ICMART) world report: assisted reproductive technology 2003. Fertil Steril 95: 2209-2222.
4. Kashir J, Heynen A, Jones C, Durrans C, Craig J, et al. (2011) Effects of cryopreservation and density-gradient washing on phospholipase C zeta concentrations in human spermatozoa. Reprod Biomed Online 23: 263-267.

5. Rienzi L, Vajta G, Ubaldi F (2011) New culture devices in ART. Placenta 3 : S248-S251.

6. Jain JK, Paulson RJ (2006) Oocyte cryopreservation. Fertil Steril 86: 10371046.

7. Gadea J, Molla M, Selles E, Marco MA, Garcia-Vazquez FA, et al. (2011) Reduced glutathione content in human sperm is decreased after cryopreservation: Effect of the addition of reduced glutathione to the freezing and thawing extenders. Cryobiology 62: 40-46.

8. Twigg J, Irvine DS, Houston P, Fulton N, Michael L, et al. (1998) latrogenic DNA damage induced in human spermatozoa during sperm preparation: protective significance of seminal plasma. Mol Hum Reprod 4: 439-445

9. Shekarriz M, DeWire DM, Thomas AJ Jr, Agarwal A (1995) A method of human semen centrifugation to minimize the iatrogenic sperm injuries caused by reactive oxygen species. Eur Urol 28: 31-35.

10. Zini A, Mak V, Phang D, Jarvi K (1999) Potential adverse effect of semen processing on human sperm deoxyribonucleic acid integrity. Fertil Steril 72 496-499.

11. Zini A, Nam RK, Mak V, Phang D, Jarvi K (2000) Influence of initial semen quality on the integrity of human sperm DNA following semen processing. Fertil Steril 74: 824-827.

12. Li Z, Zhou Y, Liu R, Lin H, Liu W, et al. (2012) Effects of semen processing on the generation of reactive oxygen species and mitochondrial membrane potential of human spermatozoa. Andrologia 44: 157-163.

13. Aitken RJ (1994) A free radical theory of male infertility. Reprod Fertil Dev 6 : 19-23.

14. Aitken RJ (1995) Free radicals, lipid peroxidation and sperm function. Reprod Fertil Dev 7: 659-668.

15. Alikani $M$ (2007) The debate surrounding human embryonic stem cell research in the USA. Reprod Biomed Online 2: 7-11.

16. Alikani M, Cohen J, Tomkin G, Garrisi GJ, Mack C, et al. (1999) Human embryo fragmentation in vitro and its implications for pregnancy and implantation. Ferti Steril 71: 836-842.

17. Keefe DL, Liu L (2009) Telomeres and reproductive aging. Reprod Fertil Dev 21: 10-14.

18. Otsuki J, Nagai Y, Chiba K (2007) Peroxidation of mineral oil used in drople culture is detrimental to fertilization and embryo development. Fertil Steril 88: 741-743.

19. Smith GD, Takayama S, Swain JE (2012) Rethinking in vitro embryo culture: new developments in culture platforms and potential to improve assisted reproductive technologies. Biol Reprod 86: 62.

20. Paria BC, Dey SK (1990) Preimplantation embryo development in vitro: cooperative interactions among embryos and role of growth factors. Proc Natl Acad Sci U S A 87: 4756-4760.

21. Lane M, Gardner DK (1992) Effect of incubation volume and embryo density on the development and viability of mouse embryos in vitro. Human Reprod 7 : 558-562.

22. Gardner DK, Lane M, Spitzer A, Batt PA (1994) Enhanced rates of cleavage and development for sheep zygotes cultured to the blastocyst stage in vitro in the absence of serum and somatic cells: amino acids, vitamins, and culturing embryos in groups stimulate development. Biol Reprod 50: 390-400.

23. Bavister BD (1995) Culture of preimplantation embryos: facts and artifacts Hum Reprod Update 1: 91-148.

24. Vajta G, Rienzi L, Cobo A, Yovich J (2010) Embryo culture: can we perform better than nature? Reprod Biomed Online 20: 453-469.

25. Kashir J, Heindryckx B, Jones C, De Sutter P, Parrington J, et al. (2010) Oocyte activation phospholipase C zeta and human infertility. Hum Reprod Update 16 690-703.

26. Morgan HD, Santos F, Green K, Dean W, Reik W (2005) Epigenetic reprogramming in mammals. Hum Mol Genet 1: 47-58. 
Citation: Kashir J, Yelumalai S, Jones C, Coward K (2012) Clinician-Induced (latrogenic) Damage Incurred during Human Fertility Treatment: Detrimental Effects upon Gamete and Embryo Viability and the Potential for Epigenetic Risk. Human Genet Embryol 1:e105. doi:10.4172/2161 0436.1000 e105

Page 3 of 3

27. Haaf $T$ (2006) Methylation dynamics in the early mammalian embryo: implications of genome reprogramming defects for development. Curr Top Microbiol Immunol 310: 13-22.

28. Qiao J, Chen Y, Yan LY, Yan J, Liu P, et al. (2010) Changes in histone methylation during human oocyte maturation and IVF- or ICSI-derived embryo development. Fertil Steril 93: 1628-1636

29. Amor DJ, Halliday J (2008) A review of known imprinting syndromes and their association with assisted reproduction technologies. Hum Reprod 23: 28262834.

30. Lim D, Bowdin SC, Tee L, Kirby GA, Blair E, et al. (2008) Clinical and molecular genetic features of Beckwith-Wiedemann syndrome associated with assisted reproductive technologies. Hum Reprod 24: 741-747.

31. Hiura H, Okae H, Miyauchi N, Sato F, Sato A, et al. (2012) Characterization of DNA methylation errors in patients with imprinting disorders conceived by assisted reproduction technologies. Hum Reprod 27:2541-2548.

32. Bridges PJ, Jeoung M, Kim H, Kim JH, Lee DR, et al. (2011) Methodology matters: IVF versus ICSI and embryonic gene expression. Reprod Biomed Online 23: 234-244.

33. Ciapa B, Arnoult C (2011) Could modifications of signalling pathways activated after ICSI induce a potential risk of epigenetic defects? Int J Dev Biol 55: 143 152 